\title{
Use of a Pathfinder Optical Telescope Element for James Webb Space Telescope Risk Mitigation
}

\author{
Lee D. Feinberg ${ }^{a^{*}}$, Ritva Keski-Kuha ${ }^{\mathrm{a}}$, Charlie Atkinson ${ }^{\mathrm{b}}$, Scott C. Texter ${ }^{\mathrm{b}}$ \\ ${ }^{2}$ NASA Goddard Space Flight Center, Greenbelt, MD, USA 20771; \\ 'Northrup Grumman, Redondo Beach, CA USA
}

\begin{abstract}
A Pathfinder of the James Webb Space Telescope (JWST) Optical Telescope Element is being developed to check out critical ground support equipment and to rehearse integration and testing procedures. This paper provides a summary of the baseline Pathfinder configuration and architecture, objectives of this effort, limitations of Pathfinder, status of its development, and future plans. Special attention is paid to risks that will be mitigated by Pathfinder.
\end{abstract}

Keywords: Space Telescope, JWST, OTE, James Webb Space Telescope, Pathfinder, Risk

\section{INTRODUCTION}

The testing of JWST Optical Telescope Element (OTE) ${ }^{i}$ and Integrated Science Instrument System (ISIM) occurs as an integrated entity called the OTE-ISIM (OTIS) at the Johnson Space Center ${ }^{i i}$. In order to assure the test is successful with as few cryogenic cycles as is possible, an OTE Pathfinder is being funded by the prime contractor Northrop Grumman as an $I R+D$ funded effort. NGAS's current plan emphasizes optical checkout at cryogenic temperatures though an augmentation to the Pathfinder plan is in the process of being added to the program that would add thermal checkout and dynamics objectives.

\section{HISTORY OF PATHFINDER}

The OTE Pathfinder has its roots in the Verification Engineering Test Articles implemented on the Chandra program wherein unfinished flight optics were used to aid in the development of, and to demonstrate the integration operations and X-ray optical testing of, the critical optical components of the telescope using GSE that was uniquely designed for the purpose. A significant component of the VETA-I and VETA-II effort was the rehearsal and demonstrations of integration and testing operations. This served to significantly reduce the implementation risk of the GSE and integration and X-Ray optical testing of the flight optical assembly. Similarly, the JWST OTE Pathfinder is intended to reduce the implementation risk of the assembly, integration, and cryogenic optical test of the JWST optical assembly. A key goal for the OTE Pathfinder is to allow early checkout of the optical test procedures and test equipment to be used for cryogenic optical testing prior to their use in testing the flight hardware. This is the original key objective of Pathfinder because it significantly reduces the likelihood that the Flight OTIS will require extra JSC test cycles because of optical GSE not performing as expected. Additional unplanned JSC cryogenic cycles pose a risk to flight hardware, risks performance degradation, and have large cost and schedule implications risks, as a single cool-down and warm-up cycle is expected to take of order two months to complete. 


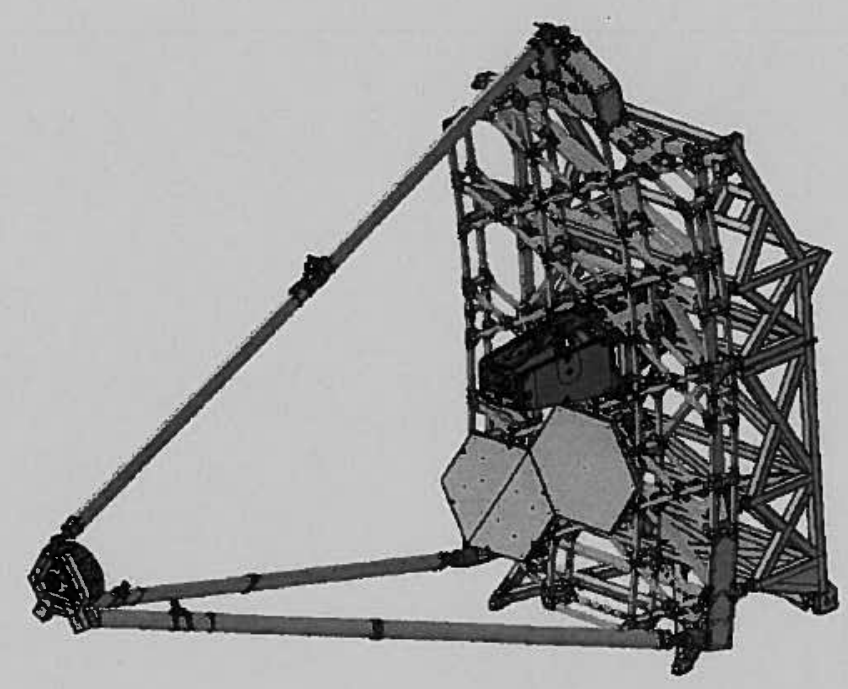

Figure 1: Pathfinder Configuration

\section{PATHFINDER DESCRIPTION}

The OTE Pathfinder configuration consists of a Center Section structure and enough Backplane Support Fixture (BSF) structure to allow for mounting, handling and shipment. Some of the Pathfinder BSF is used only for ambient handling and is removed for cryogenic testing. The OTE Pathfinder shown in Figure 1 contains a Primary Mirror Segment Assembly (PMSA) A-segment (the Engineering Design Unit - EDU) that is polished to flight requirements and coated, a PMSA C-segment spare that is uncoated and polished to approximately $100 \mathrm{~nm}$ RMS at ambient 0-g, the EDU Secondary Mirror Assembly (SMA) that is uncoated and polished to approximately $100 \mathrm{~nm}$ RMS at ambient (currently expected to be $<100 \mathrm{~nm}$ RMS at cryo) $0-\mathrm{g}$, and for a limited time, the flight Aft Optics System (AOS). The PMSAs are positioned in the A4 and C4 locations in the Pathfinder (PF) Center Section. The Pathfinder Secondary Mirror Support Structure (SMSS) is flight-like in structure, but without mechanisms engaged. The Pathfinder Center Section is without wings and only contains interfaces for the 2 PMSAs, the SMSS, the AOS, and hard-points to support ground handling. It is similar in construction to the flight Primary Mirror Backplane Support Structure (PMBSS), but without the pre-preg bleedout, process controls, and inspection data to ensure nanometer level stability at operating temperatures. It is designed to support only the optics described, and to withstand transportation loads and support the test configuration at cryo.

The Pathfinder effort at the cryogenic test facility at JSC is shown in Figure 2 and includes installation on the Hardpoint and Offloader System (HOSS), cryogenic testing without the AOS, an augmentation test of thermal GSE, and a cryogenic test with the AOS, and then egress. The first Pathfinder cryogenic test will be operated with the JSC shrouds set to achieve approximately the same temperatures on the OTE Pathfinder as will be achieved with the flight OTIS test. The second augmentation Pathfinder test will follow the basic timeline of the flight test in terms of balance points, temperatures, and flow. The last cryogenic test of the Pathfinder will have the AOS in place, but will also have the Beam Image Analyzer (BIA) in place to collect images at the Pathfinder focal surface. Due to limitations of the BIA, this second cryogenic test will be performed with the JSC shrouds set to no lower than 30K, to ensure the BIA is not driven below its cold survival temperature. The Pathfinder OTE is mounted to the HOSS using the same hardpoint connections that will be used for flight. Ballast mass is added to the HOSS to replicate the expected mass and CG of the OTIS so that the suspension system can be tuned and the isolation and damping can be exercised as it will be during testing of the OTIS; however, the Pathfinder is not intended to match the dynamic response of the OTIS. 


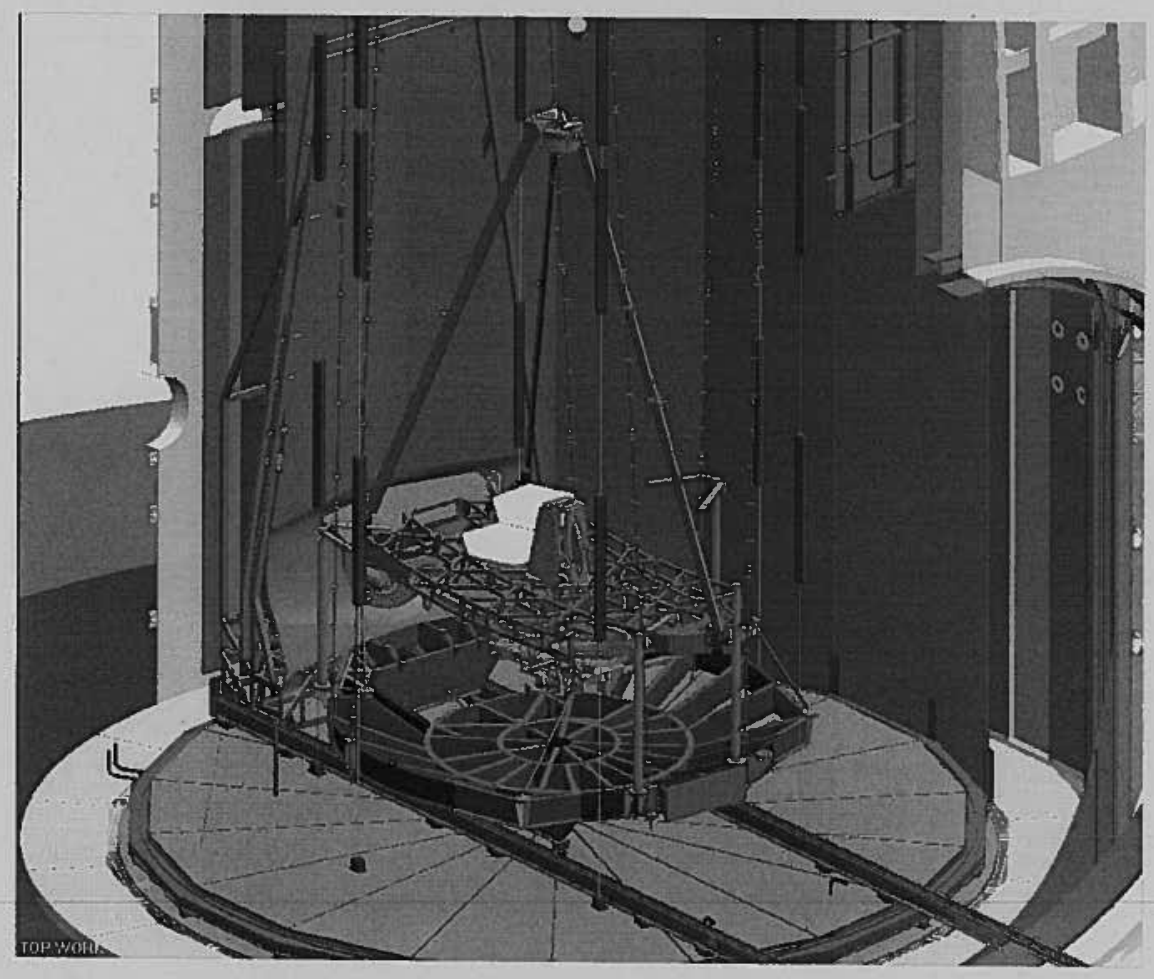

Figure 2: Pathfinder at JSC

A key objective of the OTE Pathfinder is to check out the procedures and GSE that will be used as part of the optical testing of the OTIS at JSC. To do this most efficiently, MLI is not put on the Pathfinder OTE to enable much faster cooldown and warm-up timelines than will be experienced for flight. There are also a large number of flight hardware elements that will not be replicated during the OTE Pathfinder testing, including the Center Section wings, the Backplane Support Fixture, and the ISIM and the IEC.

\section{OPTICAL OBJECTIVES}

The testing planned with the OTE Pathfinder is intended to exercise all the optical tests planned for the OTIS in at least some form. They are also intended to exercise the optical test equipment that will be used in the OTIS testiii. The relationship between the OTIS and the planned OTE Pathfinder testing is summarized in Table 1 below. The first column describes the objective of each key optical parameter that is measured during the final flight test of the OTIS at JSC. The second column describes the specific tests planned. The third column describes the optical test equipment being used for the test. The last column describes what is planned using the OTE Pathfinder. As can be seen from the last column, nearly every optical test can be checked out at some level using the OTE Pathfinder. 


\begin{tabular}{|c|c|c|c|}
\hline Parameter & Test & Optical Test Equipment & Pathfinder Approach \\
\hline \multirow{3}{*}{$\begin{array}{l}\text { Radiometric } \\
\text { Sensitivity }\end{array}$} & PM Collection Area & COCOA (reflection area) & With 2 PMSAs \\
\hline & Rogue Path & Rogue path illumination & With BIA \\
\hline & Pupil Alignment / Truant Path & Fiducial lights & With 2 PMSAs \& BIA \\
\hline \multirow{3}{*}{ WFSC } & $\begin{array}{l}\text { Plate Scale } \\
\text { (tilt of single ACF \& NIRCam) }\end{array}$ & Pass and a Half & With BIA \\
\hline & WFS\&C Demo & $\begin{array}{l}\text { Pass and a Half, COCOA, } \\
\text { photogrammetry }\end{array}$ & $\begin{array}{l}\text { With } 2 \text { PMSAs, SMA } \\
\text { \& BIA, limited to } \\
\text { PMSA and SMA } \\
\text { closed loop control }\end{array}$ \\
\hline & WFS\&C Influence Functions & $\begin{array}{l}\text { Pass and a Half, COCOA, } \\
\text { photogrammetry }\end{array}$ & $\begin{array}{l}\text { With } 2 \text { PMSAs, SMA } \\
\text { \& BIA }\end{array}$ \\
\hline \multirow{3}{*}{ Functional } & PMSA Envelope Control Limit & COCOA & Yes \\
\hline & $\begin{array}{l}\text { WF Control Signal Path (PMSA, SMA } \\
\text { motion control sign check test) }\end{array}$ & COCOA & $\begin{array}{l}\text { With } 2 \text { PMSAs \& } \\
\text { SMA }\end{array}$ \\
\hline & Fine Guidance Loop & Half Pass, Pass and a Half & Partial (no FGS) \\
\hline \multirow{10}{*}{ Image Quality } & PM to AOS alignment & Photogrammetry & With 1 PMSA \\
\hline & SMA to AOS alignment & Photogrammetry & Yes \\
\hline & ISIM to AOS alignment & Half Pass, Fiducial Lights & With BIA \\
\hline & PM RoC & COCOA, ADM & $\begin{array}{l}\text { Partial (no B3 segment } \\
\text { for monitoring) }\end{array}$ \\
\hline & low freq PM WFE & COCOA & With 2 PMSAs \\
\hline & mid frequency PM WFE & COCOA & With 2 PMSAs \\
\hline & PM conic & $\mathrm{COCOA}, \mathrm{ADM}$ & With 2 PMSAs \\
\hline & Ambient PMSA WFE & COCOA & With 2 PMSAs \\
\hline & $\begin{array}{l}\text { Multi-field PSF } \\
\text { (single ACF aperture, each SI) }\end{array}$ & Pass and a Half & With BIA \\
\hline & SI FOV Survey & Pass and a Half & With BIA \\
\hline \multirow{2}{*}{$\begin{array}{l}\text { Image Quality } \\
\text { Stability }\end{array}$} & $\begin{array}{l}\text { Thermal Distortion: PM WFE \& RoC } \\
\text { Change } \\
\text { (Figure drift of PM over temp change) }\end{array}$ & COCOA & With 2 PMSAs \\
\hline & $\begin{array}{l}\text { Thermal Distortion: Alignment Change } \\
\text { (SMA alignment drift over temp change } \\
\text { during warm up from cryo test temp) }\end{array}$ & Photogrammetry & Yes \\
\hline
\end{tabular}

Table 1: Pathfinder Testing 
All the aspects of aligning the PMSAs to the COCOA, shown in Figure 3, will be exercised during the OTE Pathfinder cryogenic test. This includes using the coarse and fine alignment subsystems and the identification and phasing, via multi-wavelength interferometry, of the 2 PMSAs. The steps involved in aligning the COCOA to the two PMSAs via imaging of PMSAs using the camera in the interferometer will also be performed, as will the camera's imaging of the fiducial lights (see section 3.4). The process of in-situ calibration using the reflective hologram will also be exercised. In addition, the incoherent illumination of the PMSA and edge detection methodology using the COCOA camera will be exercised.

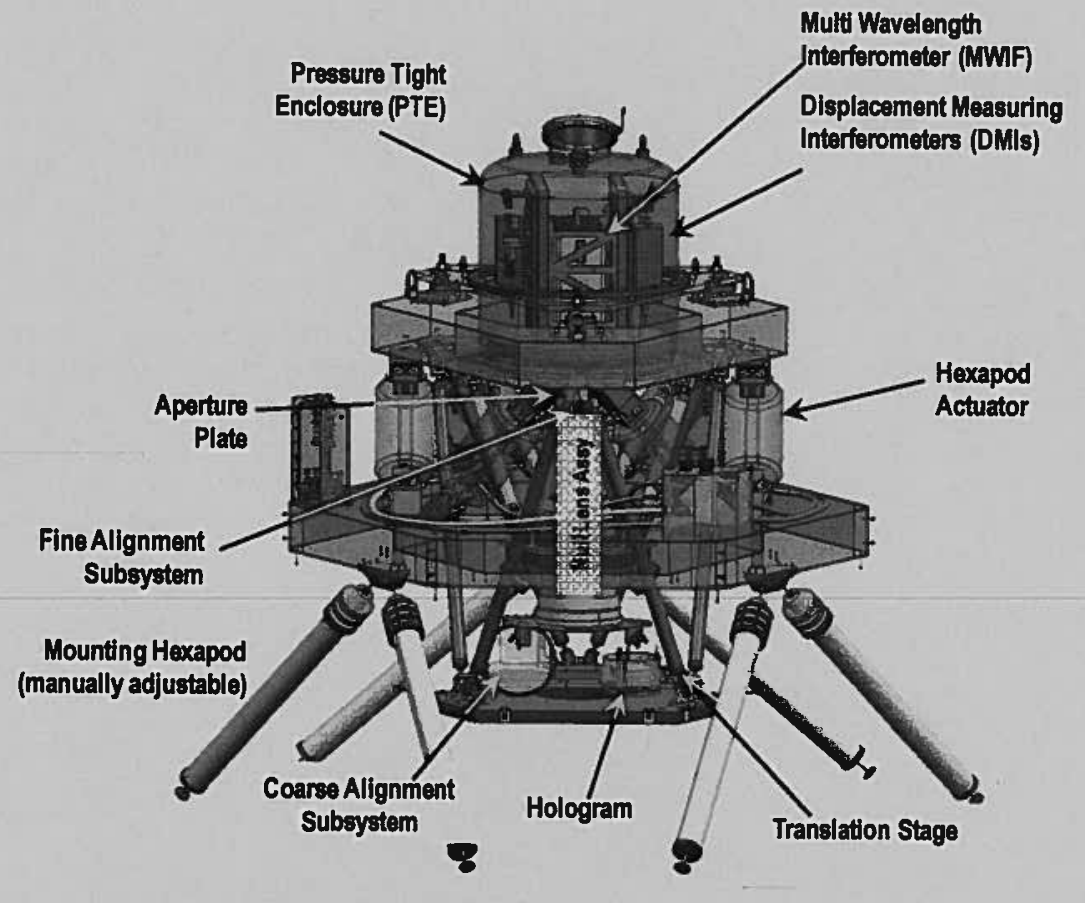

Figure 3: Center of Curvature Optical Assembly (COCOA)

The as-measured WFE of the PMSAs will be compared to what was measured at the XRCF as a means to assess the veracity of the COCOA measurement. The DMI will be used in conjunction with the interferometer to monitor RoC changes during a thermal excursion in a manner comparable to the PM Figure Stability measurement planned for the OTIS. The DMI is positioned to view the C4 PMSA, so it can be used during the Pathfinder test as well as during the OTIS test. The optical metrology for the thermal distortion test will be the same on the OTE Pathfinder as for the OTIS; however, the method to introduce the temperature change will differ due to the difference in thermal configurations. For the OTE Pathfinder test, the shroud temperature change will dominate the temperature change. Moreover, the intent of this measurement on the OTE Pathfinder is to ensure the measurement systems will work together, not to enable a comparison of predicted optical performance change to measured data, hence a quantitative expectation of observed performance will not be created. The Half-Pass test configuration shown in Figure 4 uses downward sources on the AOS Source Plate. The limiting aperture in this case is the FSM Mask. For the Pathfinder test, the BIA will take the place of the Science Instruments and provide a science-grade detector at the Pathfinder OTE focal surface on a translation stage large enough to capture the focal surface over the FoV. Through-focus images will be collected and analyzed via phase retrieval to recover the WFE. The phase retrieval algorithms will be similar to, but not identical to, those used for the OTIS testing. Focus diversity is provided by BIA motion. Pupil imaging is also provided by the BIA, necessary for phase retrieval and pupil alignment using the inward sources. In addition, a Point Diffraction Interferometer in the BIA will allow some level of comparison to the recovered WFE via phase retrieval, though the accuracy of the comparison will be coarse. 


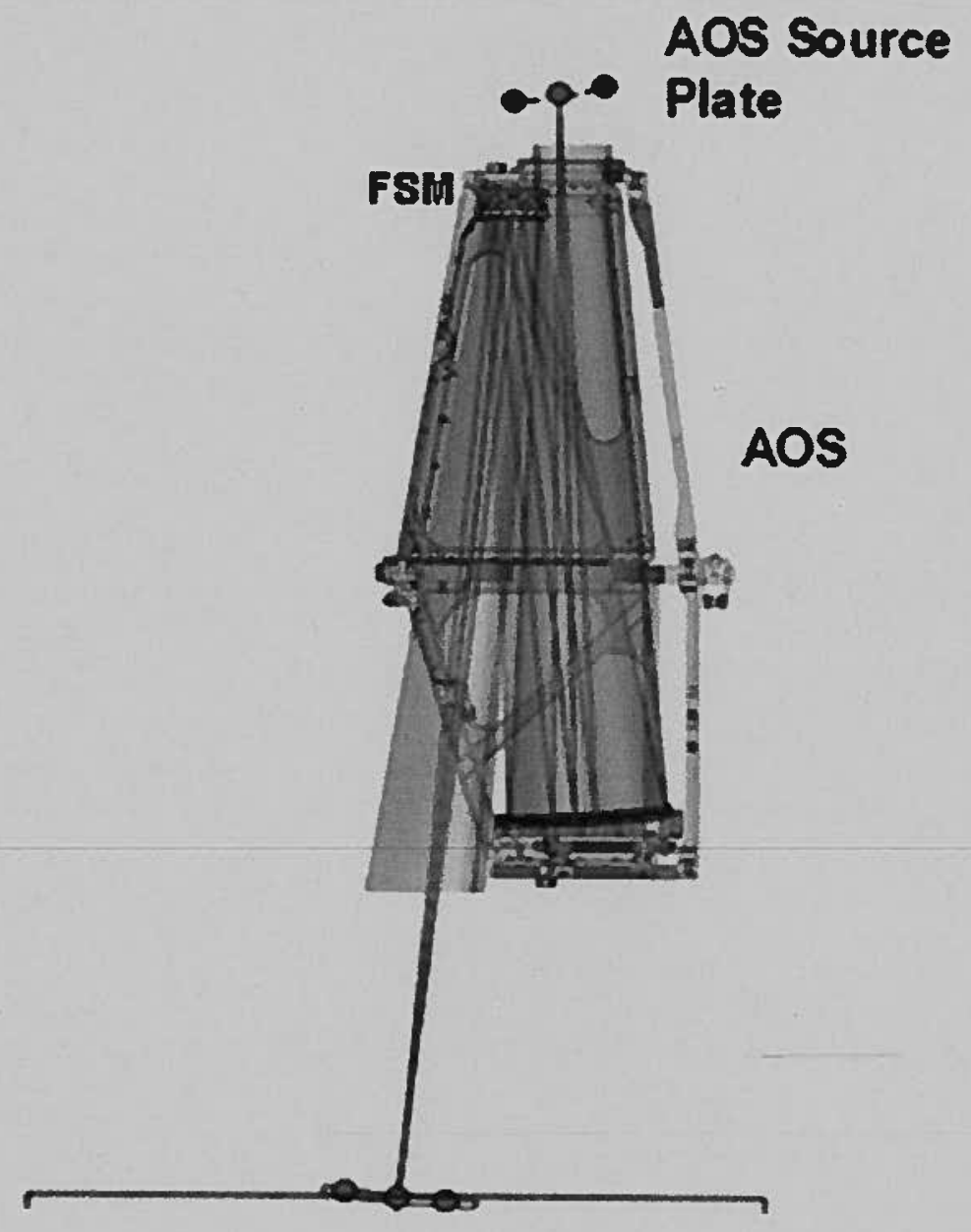

Figure 4: Half Past Test Configuration

Exercising the half-pass test enables practicing WFE recovery in the presence of the residual uncorrected WFE (up to $3000 \mathrm{~nm} \mathrm{~mm}$ ) that will also exist in the OTIS test due to the imperfect imaging at the Cassegrain focus that is an inherent part of the OTE optical design. It will also produce pupil intensity maps from the inward sources, an important input parameter to the phase retrieval algorithms. The larger range of motion of the BIA (tens of $\mathrm{mm}$ ) enables several waves of defocus, providing additional diagnostic capability.

Exercising the half-pass test will also allow for practicing determination of field tilt and image centroiding. In addition, comparing the focus, astigmatism and coma from the retrieved phase map to that expected by the source locations provides a check of the location of the sources relative to the TM. It also provides a check of the location of the OTE Focal Surface relative to the AOS interface in six degrees of freedom. Other valuable information that will be gained include the location in $1 \mathrm{~g}$ of the AOS and source plates, along with an initial practice run for imaging and image analysis in the presence of the background levels that will exist in the JSC chamber during testing.

The Pass-and-a-Half test configuration uses upward-looking sources on the AOS Source Plate. The light is collimated by the SMA and PM and that collimated light is reflected back through the OTE by the Autocollimating Flats (ACFs) and re-imaged at the OTE focal surface. The images are steered to various field locations representing the various SI fields of view by tilting the $\mathrm{ACF}(\mathrm{s})$. The limiting aperture in this case is the ACF aperture which includes partial illumination of 3 PMSAs in the flight configuration and partial illumination of the 2 PMSAs in the OTE Pathfinder 
where only a single ACF is used. During the Pathfinder test, the BIA will take the place of the Science Instruments as in the Half Pass test. Once again, phase retrieval using through-focus images recovers the WFE.

Other important optical testing includes pupil testing and cryo photogrammetry which measures alignment of the system. The testing will also check out the rogue path illumination system shown in Figure 5. During the Pathfinder test the Signal to Background ratio and overall illumination levels can be assessed whereas during the flight OTIS testing, these rays should be blocked and no illumination should be detected. This enables the Pathfinder test to demonstrate the rogue path test process and also demonstrate that a sufficient signal should be present if the OTIS rogue path mitigation is in error. It also checks the alignment of the rogue path sources to the OTE under cryogenic conditions.

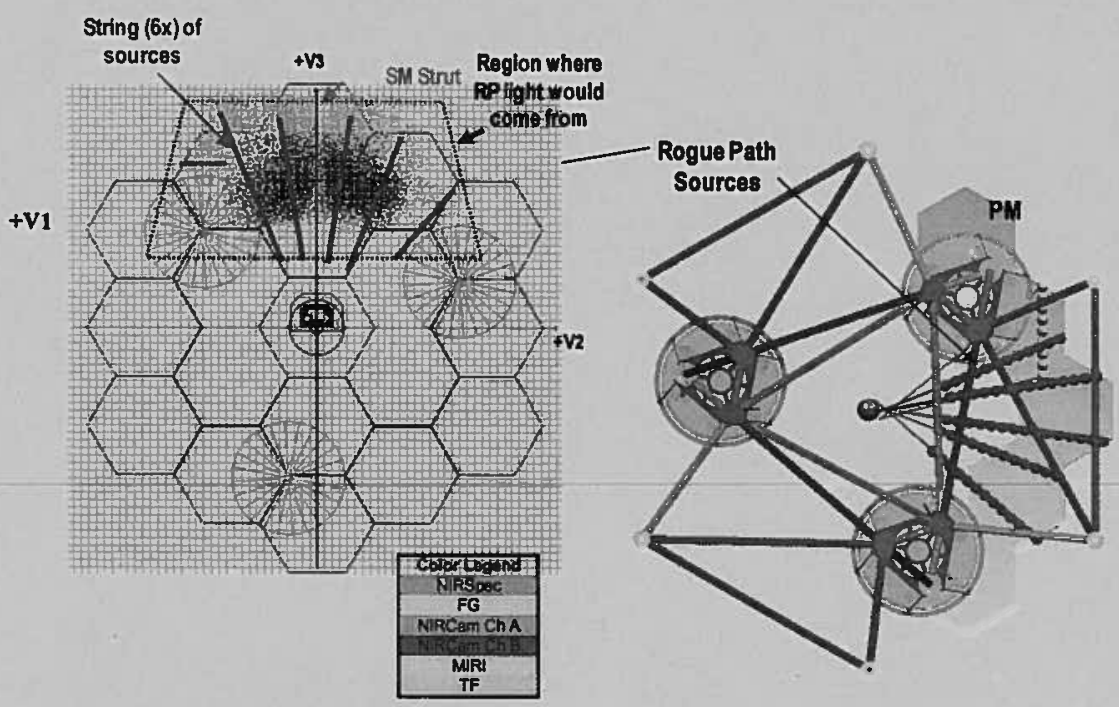

Figure 5: Rogue Path Configuration

The Pathfinder testing will also check out the cryo position metrology which consists of an Absolute Distance Meter (ADM) and the Photogrammetry system, which includes targets, cameras, rotating mechanisms and data collection and processing.

\section{DYNAMICS}

The risk reduction aspects of the Pathfinder testing at JSC as it applies to dynamics is entirely within the realm of ensuring that viable optical data can be obtained. To that end, the JSC test configuration will have the suspension system isolators and TMDs and the HOSS damper, plus some representation of the shorts to ground provided by the cables and the HOSS cooling lines. Ballast mass is added to the HOSS to replicate the expected mass and CG of the OTIS so that the suspension system can be tuned. Successfully collecting optical performance data with the interferometer in the COCOA during the first Pathfinder cryo test, and then with the Pass-and-a-half test during the second Pathfinder cryo test, will provide the desired risk reduction associated with dynamics effects on the optical measurements. 


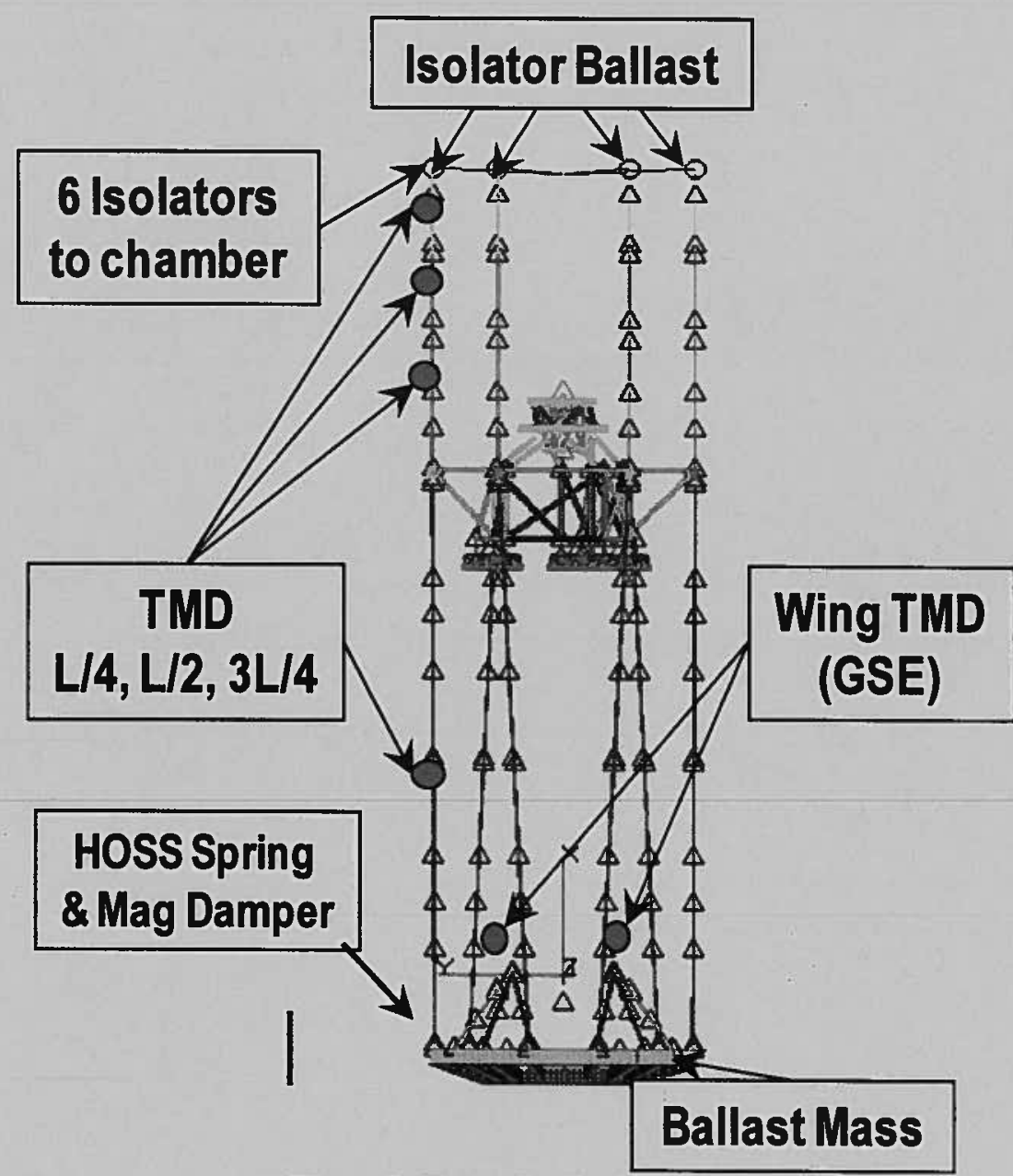

Figure 6: Dynamics Configuration

Before the OTE Pathfinder test, a dynamics model will be run using the input PSDs measured at JSC during its certification, the model of the test configuration representing the design as implemented on the JSC chamber, and a dynamics model of the OTE Pathfinder to provide an expectation for the magnitude of the vibration in the optical measurements. In addition, planning is ongoing to add cryo accelerometers and stingers to help check out the dynamics and also to address the cryo performance of the flight dynamic system.

\section{THERMAL}

The thermal configuration of the OTE Pathfinder will be quite different than for the OTIS test. Most notably, the ISIM and IEC will not be present, nor will the TMS hardware including the ISIM radiators, +/-V2 radiators, PTR and the MLI and SLI closeouts of the OTE. What will be present are the 2 non-flight PMSAs (one uncoated), non-flight SMA (uncoated), and the AOS. The flight FSM de-contamination heater will be in place and used during warm up. The method for warming up the Tertiary Mirror and SMA is undefined for the flight OTIS test, but will be replicated for the Pathfinder test at JSC. The PMSA warm-up method has been defined for the flight OTIS test. Its implementation for the Pathfinder OTE will be similar, using the OTIS decontamination heaters and some Pathfinder-specific local blanketing.

Work is ongoing to define a thermal augmentation effort that will include development of a 3D thermal model using the PMBSS thermal model. The augmentation test will propose to test the same temperature profile planned for the flight 
test including balance points and thermal distortion. The effort will include thermal and thermal distortion model correlation efforts aimed at practicing for the flight test.

\section{OTHER CHECKOUT}

During the OTE Pathfinder operations at JSC, both inside the chamber and out, the contamination protocols planned for flight will be exercised both to protect the hardware on the OTE Pathfinder and to practice their implementation on a large contamination-sensitive structure. Contamination monitoring includes particulate, molecular, temperature, and $\mathrm{RH}$ monitoring during operations in the JSC ISO Class 7 cleanroom that include unloading the OTE Pathfinder, installing the Pathfinder on the HOSS, moving the OTE Pathfinder into the chamber, performing ambient operations, performing the operations between the two OTE Pathfinder cryo tests and during exodus from the JSC facility. Witness samples will be placed adjacent to the PM like they will be for the flight OTIS test.

Warmup procedures, using the FSM heater and any GSE decontamination heaters for the Tertiary Mirror and SMA, will be demonstrated during the OTE Pathfinder testing. Re-pressurization operations using ISO Class 5 (Class 100) gas with re-pressurization rates $\leq 1 \mathrm{Torr} / \mathrm{min}$ will also be demonstrated.

The electrical and data handling aspects of the OGSE will be the same as in the flight OTIS test and therefore will be fully exercised during the Pathfinder test. However, the science pipeline and spacecraft simulator will not be used. The handling operations during the OTE Pathfinder will be a subset of those performed on flight and will therefore act as a dry run for those operations. The OTE Pathfinder will be transported using the same container as the OTIS; it will be removed from the container using the same processes as the flight OTIS. The operations to install the OTE Pathfinder onto the HOSS will be similar to, though much reduced from, the operations to install the OTIS on the HOSS. This enables checkout of the alignment on the HOSS, and several other key alignments critical to properly configuring the OTIS. Installation of the OTE Pathfinder into the JSC chamber will use many of the same operations as the OTIS, including monitoring of critical clearances through the chamber door.

\section{SUMMARY}

The OTE Pathfinder serves to reduce the risk of implementing the testing at JSC. A key element of this is to check out installation and handling procedures and optical GSE. We are in the process of adding checkout of thermal and dynamics. This will assure the GSE intended for use will work as needed during the flight test. Based on past experience, we expect the Pathfinder to be an extremely valuable tool for reducing risk to the overall integration and test program.

\section{ACKNOWLEDGEMENT}

The authors would like to thank the very large team across the country working on the JWST OTE including but not limited to key teammates Ball Aerospace, ITT, and ATK as well as L3 SSG-Tinsley, SAO and NASA/MSFC.

\section{REFERENCES}

\footnotetext{
'Feinberg, Keski-Kuha, Atkinson, Texter, "Results, Status and Plans for the JWST OTE", SPIE Proc, (2010)

ii Feinberg et al, "New Approach to Cryogenic Optical Testing the JWST", SPIE Proc. 6265-23 (2006)

iii Atkinson et al, "Architecting a Revised Optical Test Approach for JWST", SPIE Proc., 7010-26 (2008)
} 